\title{
Evolutionary Psychology and the Problem of Neural Plasticity
}

\author{
Chuck Ward \\ Department of Philosophy \\ Millersville University
}

\begin{abstract}
Evolutionary psychology as commonly presented is committed to the view that our cognitive architecture consists of a set of genetically pre-specified, domain specific, computational modules that are adaptations to the environment of our Pleistocene ancestors. These commitments yield a picture in which the underlying computational design of the human mind is genetically transmitted while cultural variation results from differential experiential inputs being processed through this common architecture. This view has been criticized from a developmental point of view. This paper develops some of those criticisms specifically as they relate to the plasticity of neural structures and their responsiveness to social interactions. In best case scenarios the confirmation of adaptive explanations involves identifying the specific causal mechanisms of selection. This is illustrated in examples from ecological genetics. This is not possible in the case of evolutionary psychology. Instead claims that certain computational modules evolved as adaptations in the ancestral environment are supported by their cross-cultural occurrence in modern populations. However, evidence suggests that behavior itself, and cultural practices, are factors that influence the development of neural structures and the cognitive processes they instantiate. So while genes are playing a role in the development of the brain, they do not really encode its neural architecture. When selection favors one set of neural characteristics over alternatives, the genes that played a role in the development of those structures are passed on. But this does not guarantee replication of the structures themselves. What is being selected? Not genes, but organisms with certain neurological and behavioral tendencies in particular environments. Variation in the genetic determinants of neurological structure is not a necessary condition for natural selection to act on behavior. The necessary condition, as Darwin originally put the point, is that traits are heritable. Certainly heritability implies some genetic transmission between generations. But heritability of neural structure requires more than a genetic determinant because neural structures are so plastic. Some regulation of the experiential environment in which those genes act is also necessary. This suggests that an adequate account of the evolution of behavior requires a multi-level approach that recognizes that gene action and social behavior are related by a kind of causal reciprocity. Such an account would be quite different than the evolutionary psychologists' model of culture being layered over the top of an underlying cognitive computer that is genetically propagated.
\end{abstract}

\section{Introduction}

Recent work in evolutionary psychology is organized around a shared set of principles regarding the nature and design of the human mind. Within this research framework the mind 
comprises a set of domain-specific, computational modules, each able to perform a specific cognitive task, and each of these modules is a genetically specified adaptation to the environment of our Pleistocene ancestors (Pinker 1997; Tooby and Cosmides, 2005). These principles have been criticized on developmental grounds (Karmiloff-Smith, 2000; Buller and Hardcastle, 2000; Durpé, 2001). The basis of such criticisms is the phenomena of neural plasticity. Evidence is accumulating that neural structures and patterns of neural connections are constantly changing over the life of organisms in response to environmental conditions, individual experience, and the behavior of the organism itself. This fact is taken, by the critics of evolutionary psychology, as a challenge some of the above tenets. In this paper I will examine the basis of the developmental criticisms of evolutionary psychology along with some responses that have been offered. I will then develop further a criticism sketched by John Dupré (2001) that specifically appeals to developmental plasticity related to culturally mediated behavior. I will argue that phenomena such as these pose problems for the kind of evidence typically used to support the claim that cognitive modules are genetically pre-specified adaptations to an ancestral environment.

In the following sections I will (1) outline the central claims that constitute the evolutionary psychology research program; (2) analyze the arguments advanced for the claim that specific cognitive modules are adaptations to the ancestral environment; (3) contrast this with the way adaptive hypothesis have been supported in other contexts, specifically ecological genetics; (4) describe some recent work on neural plasticity; (5) examine some recent developmental criticism of evolutionary psychology that appeal to neural plasticity in general; and (6) develop the criticism that focuses specifically on culturally mediated behavior. 


\section{The Evolutionary Psychology spin on cognitive modularity}

Evolutionary psychology is committed to a number of specific theses about the nature mind and its evolution (see Pinker 1997, p. 21; Tooby and Cosmides 2005, pp. 16-18).

(1) The Computational Thesis (CT): Brains are computational systems. This means that cognitive processes (and mental processes generally) are to be understood as information processing, the conversion of input to output. To quote Tooby and Cosmides,

The brain's evolved function is to extract information from the environment and use that information to generate behavior and regulate physiology. Hence, the brain is not just like a computer, it is a computer. (2005, p. 16).

Psychology/cognitive science undertakes to explain behavior by discovering the programs that facilitate these computational processes. We are not necessarily conscious of the operation of these programs, though their operation may be accompanied by conscious states. For example, the brains of children process information about language in very specific ways to produce competent language use. The children are conscious of their use of language, but they are not conscious of the way their brains processed the input that lead to that ability. The computational thesis per se is not specific to evolutionary psychology. In fact it is the predominant view within cognitive science generally - though there is considerable debate about the nature of computational processes (i.e. the Representational Theory of Mind/Symbolicism vs. Connectionism vs. a Dynamic Systems Theory approach to modeling computational systems vs. Distributed Cognition approaches).

(2) The Modularity Thesis (MT): Neural structures instantiate a modular set of cognitive processes. The various cognitive tasks that the brain performs are not all accomplished by one central processor. Rather, each cognitive task is carried out by a specialized "module" 
implementing a set of computational algorithms sufficient for its task. A module is a discreet computational unit, presumably realized in some discreet neural circuit (which isn't to say the some neurons cannot be part of more than one such circuit). The general modularity thesis is also a widespread view within cognitive science.

(3) The Domain Specificity Thesis (DST): These cognitive modules are domain specific, which is to say that they are designed to carry out some task in a specific domain (e.g. face recognition, mate selection, or cheater detection). This idea is distinguishable from the general modularity thesis (i.e. item (2) above), but for evolutionary psychologists they are tightly linked. The various cognitive tasks are carried out by discreet modules rather than one central processor (i.e. the MT). Moreover modules do not employ one set of general computational or logical principles. The very point of modularity is so that the various modules can be designed to carry out specific tasks without having to worry whether the processes or principles used would work well in the performance of other tasks. So the programs do not need to adhere to general logical principles, as long as the rules they do employ work in the domain in question.

(4) The Genetic Specification Thesis (GST): Our neural (and hence cognitive) architecture is the product of complex, evolved genetic developmental programs. The way that genes produce cognitive modules is by regulating the development of the brain and its neural wiring. Natural selection has crafted developmental programs that can reliably produce (in environments such as ours) physical properties in the brain that implement the domain specific programs described above. These developmental processes take place over an extended period of time and are often designed to be sensitive to environmental inputs which can activate (or deactivate) some gene thereby initiating its regulatory effect. 
Evolutionary Psychologists clearly consider our basic cognitive architecture to be the product of natural selection acting on genes that code for those features. This has lead to charges of genetic determinism, and evolutionary psychologists are quick to reject that charge. They are keen to point out that genes do not regulate brain development in such a way as to produce rigid behavior. They claim to be well aware of the complexities of development in general and brain development in particular. Genes do not cause behavior directly. They do so by regulating brain development (Tooby \&Cosmides 2005; Pinker 1997). And development involves both genes and the environment in which they act. Tooby and Cosmides write: "These elements [i.e. genes] are transmitted from parent to offspring and together with stable features of the environment, cause the organism to develop some design features and not others.” (2005 p. 21) This recognition of the role of environment constitutes, in their minds, a transcendence of the “old” nature vs. nurture debates or concepts. Nevertheless it is clear that evolutionary psychologists consider our cognitive architecture to be specified in the genetic code. While Steven Pinker recognizes that "[ $\mathrm{t}] \mathrm{he}$ genetic assembly instructions for a mental organ do not specify every connection in the brain as if they were a wiring schematic for a Heathkit radio" (1997, p. 35) he can also assert that '[t]he modules' basic logic is specified by our genetic program” (1997, p. 21). The assumption is that the evolution of the genetic programs takes advantage of the reliably stable features of the developmental environment.

(5) The Adaptationist Thesis (AT): These domain-specific cognitive modules are adaptations to the environment of human populations of the Pleistocene. This is often referred to as the environment of evolutionary adaptatedness (EEA). "Our modern skulls house Stone Age minds" (Cosmides and Tooby, 1997). They are designed by natural selection to generate specific types 
of behavior in certain environmental contexts, namely the context of the EEA. The results can at times be less than optimal in our present environment insofar as it differs from the EEA.

Research in evolutionary psychology seeks to discover these evolved, domain-specific cognitive modules, these "human universals" that make up human nature. These modules are genetically determined and species typical; though there will be some evolved modules that are not universal but are, rather, present in some portion of the population due their having a frequency dependent selective value. Evolutionary psychologists are quick to point out that this view does not imply rigid, genetically determined behavioral patterns. They are talking about the way information is processed by the brain. Given different inputs we should expect different outcomes. So individual and cultural variation can be arise due to variation in social environments and individual experience. But the universal aspects of human nature will be found in all normal individuals and serves as the basis for any variation that might arise. The picture is one in which the underlying computational design of the human mind is genetically transmitted while cultural variation results from differential experiential inputs being processed through this common architecture.

\section{Identifying Adaptive Modules}

Generally speaking evolutionary psychologist work at the level of the program or software. They seek to describe the computational principles employed by the adaptive modules in processing input and generating behavior. It is assumed that the module is realized in some neural network, but evolutionary psychologists don’t generally aim to identify specific brain structures involved in cognitive processing. Another methodological point is that evolutionary psychologists describe their work as a kind of reverse engineering. Once an evolved adaptive 
module is identified, then the principles employed in the solution can be worked out. The first step in this sequence is the identification of an adaptive module. The evidence invoked in support of a claim that some behavioral pattern is caused by an evolved, domain-specific adaptive module is two-fold. First the behavioral pattern must be fixed in the human population (or, alternatively, present in a definite proportion of the population in the case of frequencydependent adaptations). Second the behavior must constitute a case of complex functional design. The first step is not sufficient alone since there are numerous cases of species typical behavior that did not evolve as an adaptive solution some problem in the EEA. Tooby and Cosmides cite the use of written language. Learning a spoken language, on their account (following Pinker and Bloom) is the result of an adaptive neural program designed for that function. On the other hand "[t]he ability to read and write are by-products of adaptations for spoken language, enabled by their causal structure” (Tooby and Cosmides 2005, p. 26). Several evolutionary psychologists have argued that we can have "design evidence," i.e. evidence that some feature is an instance complex functional design, prior to identifying the selective forces that shaped the feature (Tooby and Cosmides 2005, pp. 27-8; Pinker and Bloom 1992, pp. 454-

5). The schematized argument that a module is an adaptation to the EEA runs as follows:

1. Natural Selection is the only available explanation for complex functional design.

2. The brain's cognitive architecture exhibits complex functional design (this claim can be applied globally or to a particular cognitive faculty, e.g. language use, see Pinker and Bloom, 1992)

3. So the elements of that brain's cognitive architecture are products of natural selection, i.e adaptations (in the neo-darwinian sense).

4. Evolution by natural selection takes a very long time, so the adaptations in our brain/cognitive architecture are adaptations to the EEA for human beings, i.e. the environment of our Pleistocene ancestors.

Once the conclusion is reached the job of reverse engineering can begin, that is the task of identifying the design features of the module and its adaptive significance. This is initially an 
abductive process: a computational model (or set of alternative models) must be developed as hypothetical solutions to some adaptive problem faced by our Pleistocene ancestors (e.g. kin detection/inbreeding avoidance). Development of these models should be informed by the kind of information available to our ancestors in the EEA. Then a variety of techniques might be used to determine whether or not modern humans process environmental information of that sort in the way the model proposes (Tooby and Cosmides 2005, p. 28) The basic logic of the argument seems to be something like this: this computational process would be adaptive in the EEA; modern humans seem to employ this process; therefore a computational module that carries out this process probably evolved during the Pleistocene.

\section{The Confirmation of Adaptive Hypotheses}

Attributions of adaptiveness are pretty standard modes of explanation in biology. But how are such hypotheses confirmed? The standard or ideal way in which adaptive explanations get confirmed is the painstaking process of identifying the specific agent of selection - i.e. the feature of the environment that exerts a selective pressure - and some demonstration that the proposed adaptive trait actually leads to reproductive advantage in the relevant environment. I will illustrate this process briefly with a simple example that does not involve brains, or even behavior: polymorphism in the shell color of snails.

'Polymorphism' refers to a situation in which there are multiple phenotypes, with respect to some general characteristic, in a population. "Stable polymorphism" refers to the situation in which a given population indefinitely continues to exhibit the diversity of phenotypes, as opposed to cases in which the diversity is a temporary step along the way toward one type becoming fixed in the population and the others fading out. An example of stable polymorphism 
in human beings is hair color. In the mid-twentieth Century, a group of biologists at Oxford sought to apply the framework of the neo-Darwinian synthesis to the explanation of stable polymorphism. There are plenty of examples of species divided into different breeding populations, each showing stable polymorphism for some trait or traits but where the variants occur in different frequencies in different populations. The traditional explanation for such a state was random drift occurring in traits with no adaptive significance. Since having one or another version of the characteristic afforded no advantage or disadvantage, the multiplicity would continue indefinitely.

The Oxford ecological geneticists, however, sought to overturn this interpretation. For this purpose, two members, A. J. Cain and P.M. Sheppard, conducted research on the common land snail Cepaea nemoralis which is polymorphic for a number of characteristics including shell color, which could run from red to brown to yellow, and banding pattern which runs the range from no bands to quite a lot. In 1949, they surveyed some snail populations around Oxford that occupied various habitats - fields, woods, hedgerows, etc. - and found correlations between habitat and shell type frequencies. For example, the darker shells predominated in the woods and the lighter shells predominated in the meadows (Cain and Sheppard,1950). They argued that the best explanation of these results is that natural selection is determining shell ratios. In making this argument they provided reasons for rejecting direct environmental causes and random genetic drift as alternative explanations. And they conjectured that the mechanism of selection was a combination of physiological advantage of certain gene combinations and predation pressures.

The work of Cain and Sheppard was being scrutinized by another snail fancier, French biologist Maxime Lamotte. He had also been researching polymorphism in C. nomoralis and 
while he agreed (with some reservation as to the generalizability of the conclusion) that their results offered "good evidence" that natural selection was effecting shell type distributions in the observed populations (Lamotte, 1951), he contended that it did not constitute evidence for their more specific hypothesis about the mechanism of selection. He argued that more specific information regarding the predation patterns in those population was needed. Sensitive to Lamotte's contention, Cain and Sheppard conducted a series of further studies and published papers aimed at advancing their explanatory hypothesis. When they indeed obtained results showing that snails with different shell types were eaten by thrushes at different rates consistent with their hypothesis, a new paper was published presenting these results as confirmation of their hypothesis. Lamotte, now impressed, agreed that the results constituted some evidence for their hypothesis, but still withheld the label of confirmation. He needed more, for example, whether the measured differences in predation were really enough to account for the measured differences of frequency, whether thrushes were the most significant predator, and whether predators relied on sight to find their dinner. Lamotte's own work as well as the continuing research of Cain and Sheppard aimed at figuring out ways to answer these questions. As details were added to our understanding of the ecological processes, the case for the hypothesis grew as well.

The pattern of confirmation that developed over time in the work of Cain and Sheppard (and Lammotte) was seen again a few years later in Kettlewell's famous work on industrial melanism in the Peppered Moth (Kettlewell 1973). While it might seems obvious that the speckeled-white moths would be easier to see than the dark moths on the dark tree trunks, and so eaten by birds more often, Kettlewell conducted elaborate release-recapture and aviary experiments to confirm that differential predation was in fact taking place. What these case 
studies show is that even in fairly simple cases of ongoing selective mechanisms, evidence is difficult to obtain.

Getting direct evidence of selective pressures and reproductive advantage of variant types in cases of past evolution is near impossible. This makes things very difficult for evolutionary psychologists in terms of confirming their hypotheses that certain cognitive traits were adaptive in the Pleistocene. So instead of the kind of direct confirming evidence of the sort describe for ecological genetics, evolutionary psychologists invoke the kind of argument outlined above. Instead of confirming the presence of the phenotype in the ancestral population and the reproductive advantage it had over competitors, the type is demonstrated in modern populations. The traits being an adaptation to the ancestral environment is proposed as the best explanation for its universal presence in the modern population. And this claim is supported by the traits being an example of a complex adaptive design.

But there problem: neural plasticity. Evidence is mounting from research in developmental cognitive neuroscience that the specific structures in the adult human brain are the result of the brain's own response to environmental inputs. In some instances these responses themselves may be shaped by cultural practices that potentially yield adaptive results. This introduces the possibility of changes in species typical neural structures that are not the result of modification in the genome and that have been introduced more recently than the EEA.

\section{Neural Plasticity and the Ever Developing Brain}

Despite their recognition of the importance of development in the production of neural structures, evolutionary psychologists have received criticism that charges their view with ignoring some significant features of brain development. In particular it has been suggested that 
the phenomena of neural plasticity raises doubts about some of the theses that make up the evolutionary psychology framework. Neural plasticity refers to changes in the functional organization of the brain in response to sensory inputs and the brains own activity patterns. Recent work has suggested that specific experience plays an important role in the determination of our neural wiring. This calls into question just how much of the computational architecture of our brains is specified in the genetic program.

For a long time the standard view about brain development held that humans were born with all the neurons they would ever have and that postnatal changes to the brain primarily involve cell death. Over the past thirty years or so this view has been replaced with a view of the brain as a dynamic system that undergoes a host of structural changes over the course of our lives. Many of these changes are responses to the experience of the individual.

The phenomena of neural plasticity has been part of neurological theories for over a hundred years. William James was already discussing such things in his Principles of Psychology of 1890. There he posited habit as a central principle of mental phenomena and discussed patterns or currents of nervous activity as the physical aspect of habit.

If habits are due to the plasticity of materials to outward agents, we can immediately see to what outward influences, if to any, the brain-matter is plastic. . . . The only impressions that can be made upon them are through the blood, on the one hand, and through the sensory nerve-roots, on the other; and it is to the infinitely attenuated currents that pour in through these latter channels that the hemispherical cortex shows itself to be so peculiarly susceptible. The currents, once in, must find a way out. In getting out they leave their traces in the paths which they take. The only thing they can do, in short, is to deepen old paths or to make new ones; and the whole plasticity of the brain sums itself up in two words when we call it an organ in which currents pouring in from the senseorgans make with extreme facility paths which do not easily disappear. (James 1890)

Those pathways that are traveled frequently become deeper and more apt to be traveled again. James’ contemporary, neuroanatomist Santiago Ramón y Cajal hypothesized the increased 
branching of neurons, increasing the number of connections between them, as a result of training and experience (Rosenzweig 1996). This line of thought was translated into somewhat more contemporary terms involving neuron firing by Donald O. Hebb in 1949. Hebb coined the term use-dependent plasticity and hypothesized that the strength of synaptic connections is increased as two neurons (one pre-synaptic neuron and one post-synaptic neuron) fire together. This "strengthening" increases the efficiency of transmission of activity in the future. Starting in the early 1960s experimental work began confirming this hypothesis (Rosenzweig 1996, 2003; Mohammed et. al. 2002; Elbert et. al. 2001). As research continued neuroscientists used a range of measures to demonstrate physical changes in the brain as a result of specific types of experience, for example the enlargement of specific parts of the sematosensory cortex that map body activity in response to changing behavioral patterns. Even more recently, beginning in the 1990s, evidence is beginning to accumulate that even the adult human brain can add new neurons to itself (Schwartz and Begley 2002; Rosenzweig 2003). Work in this area is reversing what had been orthodoxy in mid-twentieth century neuroscience, namely the view that the number of neurons and the basic organization of the brain is fixed shortly after birth (Schwartz and Begley 2002, p. 167).

On the whole the brain is proving to be a very dynamic system in which structural and functional organization is the outcome of its own activity. Of particular importance in the present context are organizational changes that result from culturally mediated behavioral patterns. One area that has received a lot of attention is musical training. Some research has focused on immediate expansion of the areas of the sensorimotor cortex that represent and control the movement of the fingers and hand (see for example Pascual-Leone 2001). Other work tracks larger changes in the brain, such as growth in the corpus collosum that sends signals 
between hemispheres (Schlaug 2001). Research has also revealed some correlation between musical training in children and improved cognitive abilities in visual-spatial, verbal and mathematical domains (Schlaug, et. al. 2005; Asbury and Rich 2008). While it may be too early to draw strong or sweeping conclusions from this research, what is of interest is the suggestion that a specific culturally mediated behavior or practice can alter the functional organization of the brain and that such alterations can effect cognitive abilities outside the domain of the behaviors that produced the alterations themselves.

\section{The Developmental Challenge to Evolutionary Psychology}

Neural plasticity has been at the center of some recent criticisms of evolutionary psychology. David Buller and Valerie Gray Hardcastle have argued that "we do not have lots of 'genetically specified,' domain-specific, informationally encapsulated, cognitive processing streams” (2000 p. 308). The term 'informationally encapsulated' is one they have added to the discussion and it is not found in the writings of evolutionary psychologists themselves. Buller and Hardcastle argue, however, that it is an implied and necessary assumption in the evolutionary psychologists modularity view. The idea is that the individual, domain-specific modules operate on their own kind of information and in their own fashion. To be sure the operation of the various modules is coordinated, and some modules operate in conjunction with each other. But even in cases where modules influence each other, they usually don't have access to the kind of information other modules use. (p. 309). So while the outputs of a modules may be relevant to others, the operations happening within them are not.

Buller and Hardcastle offer arguments against (1) informational encapsulation and domain-specificity as well (the DST) as (2) genetic specification of modules (the GST). Neural 
plasticity enters into both arguments. Some forms of neural plasticity show a kind of informational overlap between different brain regions normally involved in different computational tasks. For example, certain areas of the sematosensory cortex normally function to produce representations of finger movement. In cases where sensory signals from the relevant digit cease (due to the severing of the median nerve that sends those signals from the digit to the brain) those regions immediately begin responding to signals from elsewhere. This phenomena is known as "unmasking" normally silent or weak synaptic inputs. And it shows that the regions were always wired to receive such signals, and perhaps always had been receiving them, but their response was primarily determined by the primary inputs. There is even some possibility of cross-modal informational overlap. Buller and Hardcastle cite the example of the recovery of orientation ability after the disruption of input from the ear to the relevant brain structure. That structure continues to carry out the orientation function. It has been hypothesized that it uses alternative inputs from the visual system. So the relevant brain structure is "designed" to process one kind of information but is capable of performing its function by processing a different kind of information. This shows, they argue, that modules can be domain dominant rather than domain specific. This represents a kind of "soft" modularity, rather than the strict and massive kind proposed by evolutionary psychologists.

Buller and Harcastle also invoke neural plasticity to debunk the genetic specificity of computational programs. They describe aspects of neural development that show that the eventual location and functional role played by neurons is a function of cell competition and cell death resulting from that competition. So the specific organizational properties of the brain are not genetically prespecified. "Instead, during development we find a diffuse proliferation of connectivity, which later brain activity, guided by interaction with the environment, sculpts into 
its final form" (p. 316). Given that the "hardware" is produced in this way, we cannot say that the computational processes they carry out are genetically specified. This does not mean that human brains all end up with different computational properties. Indeed we do have some set of species typical neurological structures and corresponding cognitive faculties that serve adaptive functions. But this is not the result of genetic programs specifying the computational properties of those structures. Rather it is the result of the very plasticity of the brain itself, its ability to respond to environmental conditions in ways that shape its own structure and computational design. "[T]he degree to which the outcomes of human brain development have been regular through some of hour evolutionary history is due to the fact that generally plastic brains have encountered recurrent environmental demands throughout that history” (317). The adaptive character of the brain is not some set of specific computational programs, but the plasticity itself. (321).

Annette Karmiloff-Smith invokes neural plasticity to make similar points against the genetic pre-specification of cognitive modules (2001). In her case she is addressing an important kind of evidence often employed to support the existence of domain specific modules, namely the phenomena of double-dissociation. This term applies to cases where two related capacities can be shown to operate independently by certain patterns of dysfunction. For example, children with Williams Syndrome (WS) are severely impaired in their ability to recognize objects, though they seem to have normal abilities in terms of face recognition. Other disorders might display the opposite effect: impaired face recognition capacity but seemingly normal object recognition capacity. This has been used to argue that there are two distinct modules in the brain that perform these two distinct functions. Karmiloff-Smith refers to research of her own and others to show that this argument breaks down under scrutiny. While WS children seem to have 
normal face recognition capacities, it has been shown that they use very different cognitive strategies to accomplish such tasks as compared with non-WS children. Similar considerations apply to the apparently normal aspects of WS children's language ability. Karmiloff-Smith uses these points to argue that even if the adult brain is a set of domain specific modules, this situation is the result of developmental processes, not genetic pre-specification. Through developmental processes the brain organizes itself into units that operate on specific inputs, and this organization is the result of the brains ability to respond adaptively to environmental demands. Karmiloff-Smith suggests that the brain comes with a number of domain relevant learning mechanisms which give rise to more localized domain specific modules over time.

The modularity view of the evolutionary psychologists has been defended against the arguments summarized above by John Sarnecki (2007). A key point in Sarnecki's analysis has to do with the contrast between domain-dominance (Buller \& Hardcastle) and domain relevance (Karmiloff-Smith) on the one hand and domain specificity (evolutionary psychology) on the other hand. Sarnecki argues that the distinction collapses in environments that remain relatively stable over time or show consistent variability (Sarnecki 2007, p. 538). In such environments natural selection can act to select individuals with specific neural structures and this yields the propagation of the genes that serve to bring about the development of such structures in such environments. So even if the existence of these neural structures depends on certain environmental inputs, they can still be adaptations, in the neo-darwinian sense, to typical environmental conditions.

Sarnecki's point is in keeping with the views of evolutionary psychologists concerning the interaction of genetic and environmental factors during development. Recall Tooby and Cosmides claim that genes "are transmitted from parent to offspring and together with stable 
features of the environment, cause the organism to develop some design features and not others.” (2005 p. 21). They recognize that genes can only be selected for the production of cognitive modules if the environmental factors relevant to their causing those modules to develop remain stable. Pinker spins this a bit differently, describing genes as taking advantage of the way the neurons in the developing brain process and respond to environmental input to bring about adaptive computational modules (1997 p. 35). This characterization corresponds to what Susan Oyama has called "standard (or traditional or conventional) interactionism" which she criticizes as still harboring an unacceptable degree of genocentrism (Oyama 2001)

\section{What About Culturally Mediated Developmental Environments?}

While the two developmental critiques outlined in the last section appeal to neural plasticity, it is of a fairly generic sort. This is what opens the door to Sarnecki's rebuttal. If we can assume some constancy in the developmentally relevant environmental factors (including the kinds of individual behaviors and experiences involved), then we can maintain a gene-centered approach to the development and evolution of neural structures. John Dupré has offered a developmental critique that specifically introduces the likelihood that environmental factors relevant to brain development have changed dramatically through cultural evolution. " [S]ince conditions under which contemporary brains develop are very different from the conditions under which human brains developed in the Stone Age, there is no reason to suppose that the outcome of that development was even approximately the same then as now” (Dupré 2001, p. 31). In this section I want to fill out this line of argument by appealing to some specific aspects of neural plasticity and examining some implications with regard to cognitive evolution. Dupré’s argument suggests significant, developmentally relevant differences between the 
contemporary environment and the ancestral environment. This view is bolstered by evidence (outlined in an earlier section) that some culturally mediated behavior can influence the development of neural structures, along with the corresponding computational properties of the brain. And this weakens the thesis that the core features of our cognitive architecture are adaptations to the environment of our Pleistocene ancestors.

Social aspects of the environment, like other aspects of the environment, place demands on the organism, i.e. conditions to which the organism responds. Some of those responses will produce developmental effects. Some of these responses can also constitute cultural practices, and for that reason they can be stable (in the sense of reliably present across generations) due to cultural transmission while still subject to significant modification. The developmental relevance of cultural practice does not challenge the modularity thesis per se. But it does constrain the possibility of having good evidence for modules being adaptations to the environment of our Stone Age ancestors.

To be sure, evolutionary psychologists do recognize that the social environment of human beings has changed since the Pleistocene. In fact they rely on this fact to explain the apparently maladaptive nature of some aspects of our evolved human nature.

Although the behavior our evolved programs generate would, on average, have been adaptive (reproduction promoting) in ancestral environments, there is no guarantee that it will be so now. Modern environments differ importantly from ancestral ones, particularly when it comes to social behavior. We no longer live in small, face-to-face societies, in seminomadic bands of 20 to 100 people, many of whom are close relatives. Yet our cognitive programs were designed for that social world. (Tooby and Cosmides 2005, p. 17).

So environmental conditions come into their story in three ways. First the ancestral environment presents the design problems that natural section solves through designing adaptations. Second the environment constitutes the conditions under which development takes place. Here we must 
be looking at stable features of the environment, features that are reliably present so that the genes can (co-) produce the designed programs. But the developmentally relevant features must (at least often) be different from those features that relate to the adaptedness of the programs. For the third role of the environment in their story has to do with environmental change. There are a number of things that have changed in our environment over time, and some of those have made our design features less adaptive or even maladaptive (e.g. our craving for fats and sugars). These changes alter the adaptive nature of (some of) our programs, but those programs are still there. So the environmental conditions necessary to developmentally produce them must still be present. This is where the genocentric or gentic determinism aspect of the story becomes apparent. The assumption is that modifications to the programs must be due to genetic mutation. The environmental changes that are recognized have no role in determining the nature of the programs. Recall Pinker’s claim that “[t]he modules’ basic logic is specified by our genetic program” (1997, p. 21).

A more balanced view would consider the possibility that those changes might also affect the developmental process in such a way as to give rise to cognitive structures quite different from those of our ancestors. Among the strongest candidates for environmental factors that effect brain development in ways that impact the brain's computational properties, and so our cognitive faculties, are culturally mediated behavioral patterns. They are good candidates because (a) there is evidence for behavior-related plasticity that impacts cognitive processes; (b) there are examples of culturally mediated behavior that falls into this category; (c) there is some indication that changes to the brain brought about by such culturally mediated behavior can effect a fairly wide range of cognitive abilities; and finally (d) culturally mediated behaviors can stabilize through cultural transmission. 
The evidence for item c above is the weakest. Perhaps the most researched example to date is musical training. Music, musical performance and musical training are certainly examples of cultural practices. There have been claims that musical training has a positive effect on linguistic ability, spatial reasoning, mathematics and other cognitive faculties that fall outside of the domain of playing music (Asbury and Rich 2008; Schlaug et. al. 2005). There is considerable evidence that musical training and regular practice/performance leads to physical changes in the brain (Schlaug et. al. 2005; Pascual-Leone 2001). Strong empirical evidence that specific neurological changes lead to improved cognitive functioning outside the domain of musicianship is still scant, but some evidence has been acquired.

While the significance of this set of cultural practices on the structure and function of the brain is still weak, the very possibility of culturally mediated restructuring of the brain throws a wrench into the evolutionary psychologists argument. Why is this? Because now we can identify a class of environmental factors that are relevant to cognitive development but may have been introduce more recently than the evolutionary psychologists EEA. It is also a class of environmental factors which can change more quickly than genomes and is in some sense under human control. If the functional architecture of our brains (and so our minds) is in significant part a product of our fairly recent cultural development, then those features are not adaptations to the environment of our Pleistocene ancestors.

Of course we are dealing with speculation here. But it is speculation built upon a growing body of evidence regarding what is possible in neural development. And it creates a gap in the evolutionary psychologists case for the adaptive significance of neural architecture being tied to the EEA. Recall the argumentative strategy used to support an adaptive hypothesis outlined above. And adaptive problem faced by our ancestors is identified; an adaptive 
hypothesis is developed regarding a computational process that might solve that problem; then evidence is gathered that this process is universal or very common in modern populations. The problem now is that the presence of a common computational module in modern populations could be due to the recurrence or maintenance of the relevant developmental social environment producing the relevant behaviors on the part of developing humans. The possibility is open to significant, adaptive changes in our cognitive architecture that come about without significant genetic change. Here the genes are not being changed in ways that allows them to exploit environmental factors to bring about adaptive effects. Rather people are (though not necessarily consciously) taking advantage of their genes by modifying the developmental environment to bring about adaptive effects.

The likely response to these suggestions on the part of evolutionary psychology can be summarized with two statements from Pinker. "[N]atural selection is the only evolutionary force that acts like an engineer, 'designing’ organs that accomplish improbable but adaptive outcomes" (1997 p. 36) and "The evolution of information processing has to be accomplished at the nuts-and-bolts level by selection of genes that affect the brain-assembly process” (1997 p. 176). Together these claims suggest the response that while cultural changes might bring about changes in our neural structures, these changes are not likely to be adaptive. If we find highly adaptive cognitive modules these must be the product of natural selection acting on the genetic programs that regulate neural development. I will accept the first of the above quotations for current purposes. The second is an expression of the genocentric view of evolution. On this view 'heredity' simply means the transmission of genes from parent to offspring, 'evolution' simply means changes in gene frequency in a population, and evolutionarily relevant cases of natural selection result in differential propagation of alleles. But there are other ways to think 
about evolution. Darwin himself defined evolution by natural selection not as the differential propagation of alleles, but as the differential propagation of heritable variations, i.e. phenotypes. The passage of a phenotypic trait from one generation to the next requires the transmission of genes, to be sure. But heritable variation need not be based on genetic variation, at least in the case of the neurological characteristics we are considering. Responsiveness of neural structures to culturally mediated behavior opens up the possibility of two populations, or even subpopulations, that do not differ genetically but do differ in terms of neurological phenotypes. These two populations could have different sets of neurological structures, each perfectly typical within a given population. These different neurological phenotypes could realize different computational processes and so different cognitive capacities. If one variant is more adaptive, then we can imagine differential reproduction between the groups. If the behavioral factors that contributed to the occurrence of the phenotypes are reliably transmitted within the groups, we can imagine cumulative evolutionary change. Natural selection can act in the absence of genetic variation. It is not acting on genes, but it is, rather, acting on individuals in virtue of their heritable traits.

This alternative view, which allows for the possibility of heritable neurological (and hence cognitive) variation without genetic variation, fits more comfortably with the developmental systems view of evolution (Griffiths and Gray 2001; Dupré 2001) than with the evolutionary psychologists' view. On this developmental systems view, natural selection acts on individuals, not genes. To have long term evolutionary effects those individuals must exhibit heritable variation, but heritability is construed more broadly than just the transmission of genes. It is, rather, the transmission of any stable developmental resource. This can include environmental factors that are causally relevant to developmental outcomes. Some species can 
have a causal role in maintaining such a developmentally relevant environmental factor across generations. In such cases this environmental factor becomes a heritable factor no less than physical genes. Culturally mediated practices seem a particularly good example of such a phenomena. And recent work on neural plasticity suggests that they are highly relevant in neural development and so highly relevant to any computational properties exhibited by our brains.

\section{Conclusion}

Evolutionary psychologists consider the species typical aspects of our cognitive architecture to be a set of genetically pre-specified, domain specific, computational modules that are adaptations to the environment of our Pleistocene ancestors. The evidence that evolutionary psychologists present for the claim that a particular cognitive module is in fact such an adaptation presupposes that the current environment is quite similar to the ancestral environment with respect to developmentally relevant factors. Some critics such as Dupré, along with others sympathetic to the developmental systems theory approach, have challenged this assumption. Recent work in developmental and cognitive neuroscience on behaviorally dependent neural plasticity strongly suggests that culturally mediated behaviors can have profound effects on the structure of our brains throughout our lifetimes. This makes it quite likely that cultural development since the Pleistocene has introduced developmentally relevant changes to our social environment. This, in turn reduces the likelihood that our developmental environment is sufficiently similar to that of our Stone Age ancestors and calls into question the assumptions made by evolutionary psychologists. 


\section{Bibliography}

Asbury, Carolyn and Rich, Barbara, eds. 2008. Learning, Arts, and the Brain. The Dana. Consortium Report on Arts and Cognition. Dana Press.

Cain, A.J. and P.M. Sheppard. 1950. "Selection in the Polymorphic Land Snail Cepaea nemoralis.” Heredity 4:275-94.

Cain, A.J. and P.M. Sheppard. 1954. “Natural Selection in Cepaea.” Genetics 39:89-116.

Cosmides, Leda, and Tooby, John. 1997. "Evolutionary Psychology: A Primer." Center for Evolutionary Psychology. http://www.psych.ucsb.edu/research/cep/primer.html. Retrieved March 2008.

Dupré, John. 2001. Human Nature and the Limits of Science. Oxford University Press.

Elbert, Thomas, Heim, Sabine and Rockstroh, Briditte. 2001. "Neural Plasticity and Development.” In Charles A. Nelson and Monica Luciana, eds. Handbook of Developmental Cognitive Neuroscience. MIT Press, pp. 191-202.

Griffiths, Paul E. and Gray, Russell D. 2001. "Darwinism and Developmental Systems.” In Susan Oyama, Paul E. Griffiths and Russell D. Gray, eds. Cycles of Contingency. Developmental Systems and Evolution. MIT Press, pp. 195-218.

James, William. 1898. Principles of Psychology.

Karmiloff-Smith, Annette. 2000. “Why Babies’ Brains are not Swiss Army Knives.” In H. Rose and S. Rose, eds. Alas Poor Darwin: Arguments Against Evolutionary Psychology. Harmony Books, pp. 173-188.

Kettlewell, Bernard. 1973. The Evolution of Melanism. Oxford: Clarendon Press.

Lamotte, Maxime. 1951. Rescherches sur la structure genetique des populations naturelles de Cepaea nemoralis L., Supplement to Bulletin Biologique de France et de Belguque, no. 35.

Mohammed, A. H., et. al. 2002. “Environmental Enrichment and the brain.” Progress in Brain Research: 138: 109-133.

Oyama, Susan. 2001. “Terms in Tension: What Do You Do When All the Good Words Are Taken?” In Susan Oyama, Paul E. Griffiths and Russell D. Gray, eds. Cycles of Contingency. Developmental Systems and Evolution. MIT Press, pp. 195-218.

Pascual-Leone, Alvaro. 2001. “The Brain That Plays Music and Is Changed by It.” Annals of the New York Academy of Sciences 930: 315-329.

Pinker, Steven. 1997. How the Mind Works. W.W. Norton \& Company. 
Pinker, Steven, and Bloom, Paul. 2001. "Natural Language and Natural Selection." In Jerome H. Barkow, Leda Cosmides and John Tooby, eds., The Adapted Mind. Evolutionary Psychology and the Generation of Cuture. Oxford University Press, pp. 451-494.

Rosenzeig, Mark R. 1996. "Aspects of the Search for the Neural Mechanisms of Memory.” Annual Review of Psychology 47: 1-32.

Rosenzeig, Mark R. 2003. "Effects of Differential Experience on the Brain and Behavior.” Developmental Neuropsychology 24: 523-540.

Sarnecki, John. 2007. "Developmental objections to evolutionary modularity." Biology and Philosophy 22: 529-546.

Schlaug G. 2001. The brain of musicians: A model for functional and structural plasticity. Annals of the New York Academy of Sciences 930: 281-299.

Schlaug G, Norton A, Overy K, Winner E. 2005. "Effects of Music Training on the Child's Brain and Cognitive Development." Annals of the New York Academy of Sciences 1060:219230.

Tooby, John and Cosmides, Leda. 2001. "The Psychological Foundations of Culture.” In Jerome H. Barkow, Leda Cosmides and John Tooby, eds., The Adapted Mind. Evolutionary Psychology and the Generation of Cuture. Oxford University Press, pp. 451-494.

Tooby, J. \& Cosmides, L. 2005. “Conceptual Foundations of Evolutionary Psychology” In D. M. Buss (Ed.), The Handbook of Evolutionary Psychology. Wiley \& Sons, pp. 5-67. 